AN IDEAL ARRANGEMENT FOR THE TREATMENT OF FEMORAL FRACTURES

\title{
EDWARD A. RICH, M.D.
}

TACOMA, WASH.

The accompanying illustration shows a practical and exceedingly satisfactory arrangement for the treatment of fractures of the femur. Hammerstein's extension table is made use of to fix the patient's body and limbs; an $x$-ray tube suspended on an adjustable cross-bar of the table is connected with the coil. An assistant can increase or decrease the amount of extension by a turn of the screw attachment, as can he also control the amount of abduction or adduction.

The operator thus equipped can make frequent use oi the fluoroscope during his manipulations and all guesswork is positively eliminated. When perfect position is secured there is no hindrance to the application of splints. Should plaster-of-paris casings be used we have the same unobstructed freedom as when applying spicas for tubercular hips. I use internal plaster splints and external splints of the same material, reaching from the axilla to the external malleolus with a steel rod incorporated for extension on the foot. When the splint is properly applied and secures muscular immobilization there is no reason why the bony fragments should be displaced.

The last three femoral fractures that have been referred to me were difficult cases, all of the patients

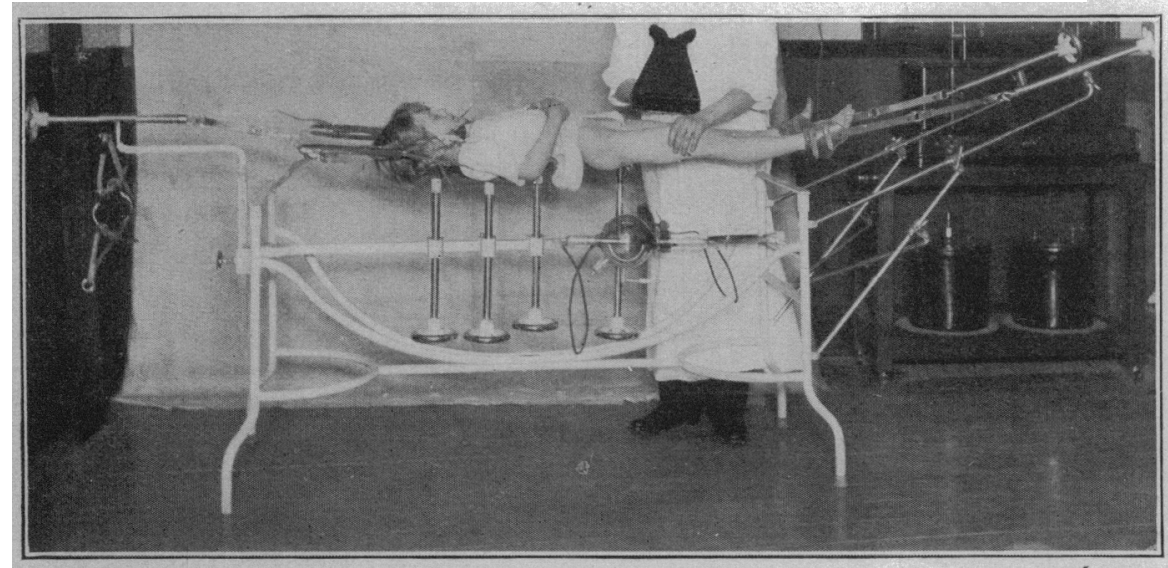

Extension table with $x$-ray attachment for the treatment of femoral fiactures by the illuminated metbod. Note the unobstructed field for application of immobilizing splints; also mode of applying extension and abduction, and case of positive reduction.
After the patient is placed in bed the tubing is connected with a drainage tube from the bladder. The Y-shaped tube $\mathrm{F}$ must be below the level of the patient lying in bed; the reservoir A filled with water, the stop-

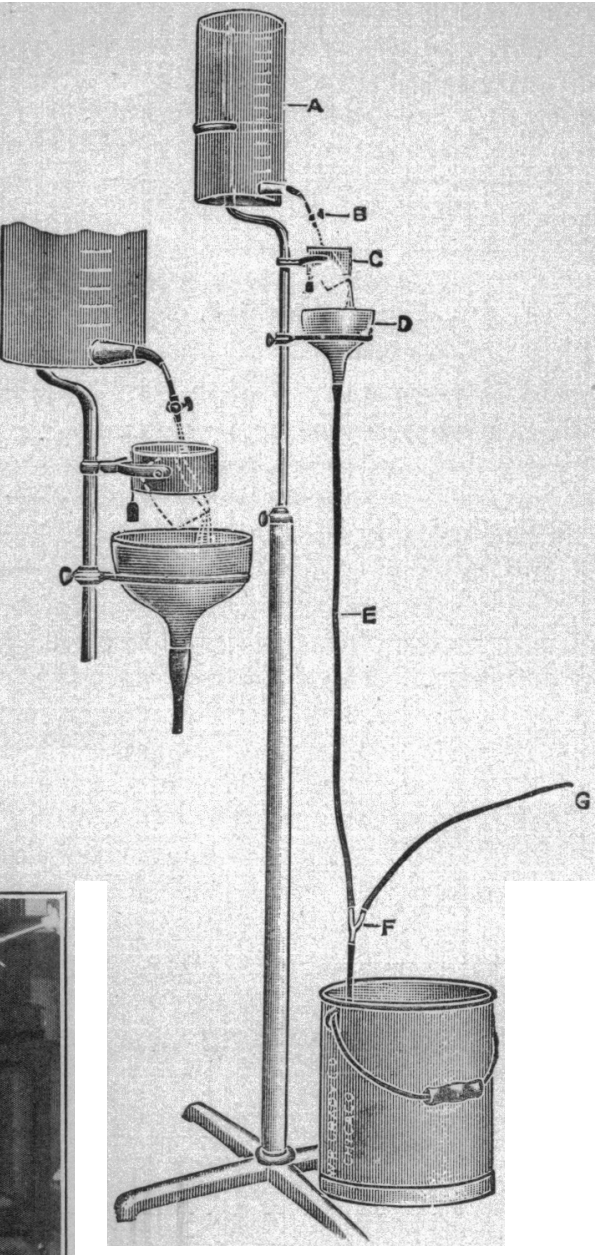

Apparatus for complete drainage of cystotomy : A, reservoil for water ; 13, stopeock for regulating the flow: $C$. cup weighted in the rear to hold in place when empty. D funnel to catch the water when the water when the cup tips on being filc with water; nects the funnel with the Y-shaped glass tule, $F$, Y-shaped glass tube; $G$, tube dratine bladder with the $Y$-shaped tube. having suffered breaks in the region of the surgical neck. The three after this method of illuminated reduction and immobilization were taken from my operating room to their homes in conveyances. Later radiographs show splendid results.

\section{NEW APPARATUS FOR COMPIETE DRAINAGE OF THE BLADDER FOLLOWING EITHER SUPRAPUBIC OR PERINEAL CYS- TOTOMY \\ LEWIS WINE BREMERMAN, A.M., M.D. CIICAGO}

Having had considerable difficulty in draining the bladder after cystotomy with the ordinary apparatus, I devised the apparatus shown by the accompanying rut, which fulfills perfectly the purpose for which it was constructed, that of perfect drainage of the bladder. cock B turned on so that the water will drop slowly into the cup $C$, which, on being filled, tilts and empties itself into the funnel $\mathrm{D}$. The water running down the long tulse $\mathrm{E}$ into the $\mathrm{Y}$-shaped tube $\mathrm{F}$ forms behind it a suction which causes a siphonage of the fluid in the bladder. This siphonage may be regulated to occur at any given interval by arranging the flow of water from the stopeock B which flows into the cup.

Usually the first twelve hours following the operation I regulate the flow so that the cup will tilt every five minutes, which is sufficiently often to keep the bladder perfectly dry. During the second twelve hours I arrange the flow so that the cup tilts about every ten minutes; during the second twenty-four hours, about every fifteen minutes.

I find this apparatus of exceptional value, particularly after prostatectomies, in which perfect drainage is exceedingly essential. Complete drainage of the bladler causes the patient to be comfortable and free from pain, 